\title{
Testing times for BSE
}

\author{
No one knows whether the diagnostic tests \\ being used to search for BSE infection in \\ Europe's cattle can reliably detect animals \\ incubating the disease. Given this limitation, asks \\ Quirin Schiermeier, what is the testing \\ programme likely to achieve?
}

$\mathrm{T}$ he spectre of bovine spongiform encephalopathy (BSE) is stalking continental Europe. France last year recorded 138 cases, and countries that had judged themselves BSE-free have been shocked out of their complacency. In Germany, the country's first home-grown BSE cases have led to two ministerial resignations; only last month, beef sales in Italy collapsed as the country's first case came to light.

Given widespread exports of potentially infective British animal feed ${ }^{1}$, the fact that BSE is now a pan-European problem should be no surprise. But for politicians who have been in denial, there is a new urgency to establish the scale of the epidemic. To find some answers, the European Union (EU) last December agreed to a programme of diagnostic testing of slaughtered cattle. Although the tests should put some figures on the extent of Europe's BSE problem, consumers anxious to avoid any risk of infection cannot rely on the testing regime. The tests being used perform well in identifying cows showing symptoms of BSE, but it is so far unclear whether they can reliably identify animals incubating the disease but showing no symptoms.

This has already caused controversy. On 6 December, Britain's Independent newspaper lambasted the European Commission for its reliance on a test that "has never been properly validated". Commission officials reacted angrily, arguing that the tests had only ever been presented as an additional safety measure. "The EU is perfectly aware of the deficiencies of the tests," responded Andrea Dahmen, spokeswoman for research commissioner Philippe Busquin. "The tests are a very useful additional measure to restore consumer confidence. However, the most vital thing is that our safety legislation is respected, especially the removal of risk materials from slaughtered ruminants."

The public was left wondering what to believe: would the tests protect them from this terrible disease? And if not, why should consumer confidence be restored? For now, those questions remain unanswerable. But despite these limitations, the EU's testing programme should allow epidemiologists to
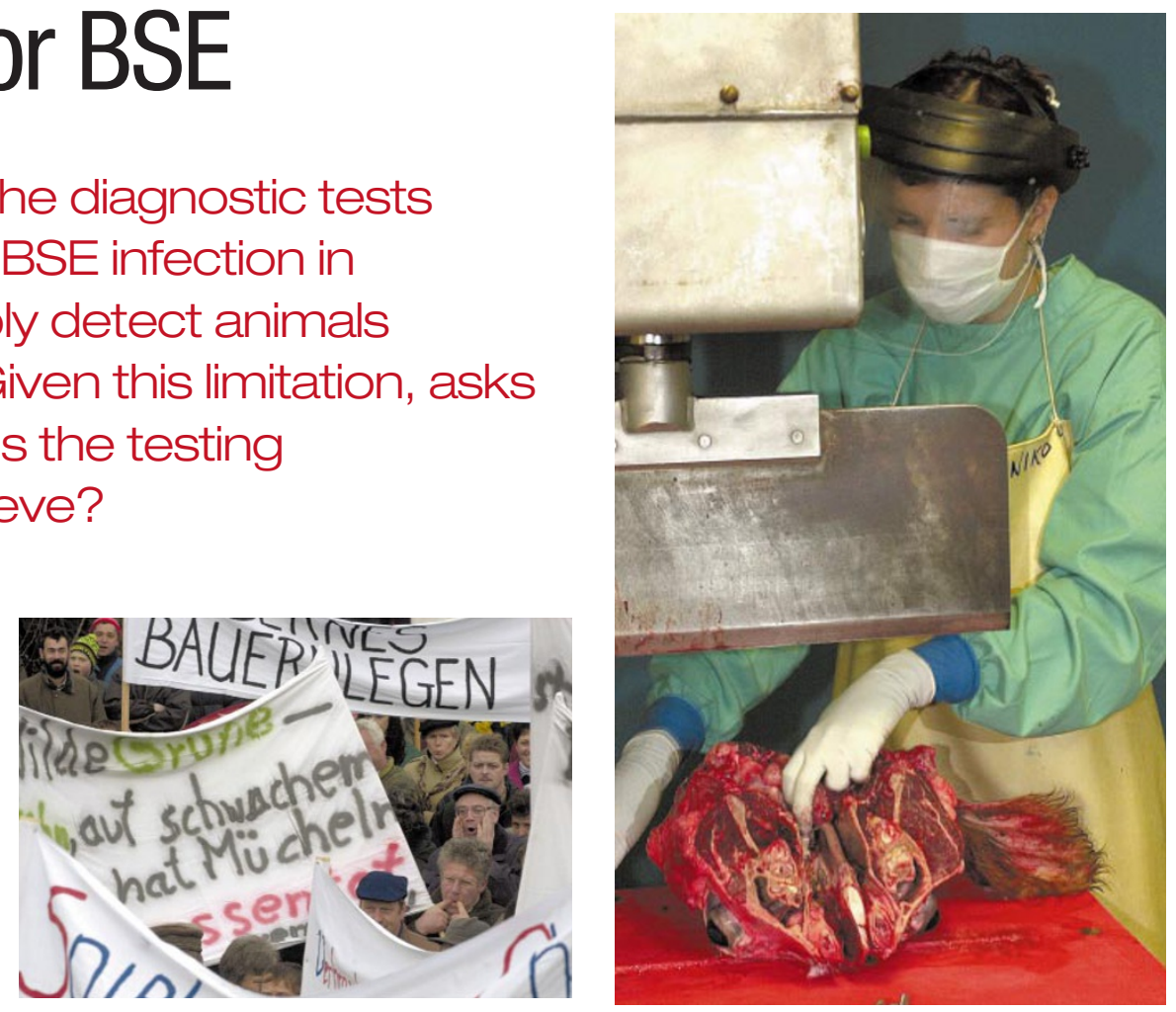

Europe under a cloud: as the BSE crisis escalates, German farmers protest about the planned culls of their herds (left) and a researcher in Spain tests a carcass for signs of the disease.

begin to assess the extent of BSE in mainland Europe. Until now, the only real certainty is that there has been widespread underreporting of the disease.

\section{Accelerated timetable}

The testing programme has two phases. From January this year, cattle that have shown suspicious symptoms must be tested with one of three approved diagnostic tests. From July, this requirement will be extended to all cows coming to slaughter aged 30 months or more. Several EU states have accelerated this timetable - France and Germany, for instance, have been testing all 'over 30-month', or OTM, cattle since

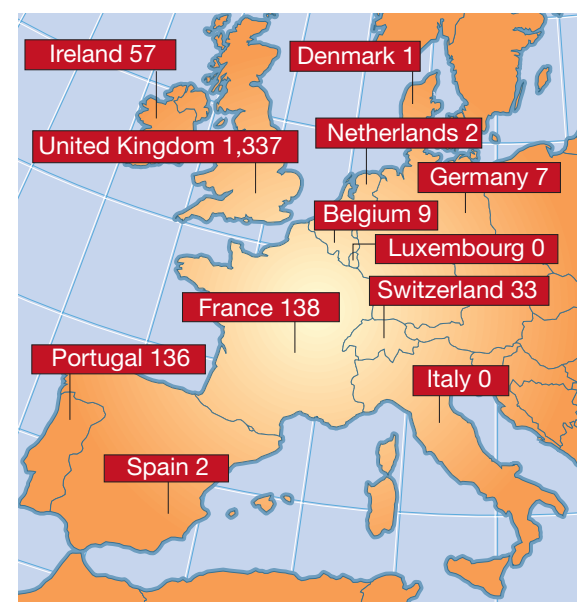

Crossing the borders: confirmed cases of BSE throughout Europe during 2000.
January; and Germany has in recent weeks extended its testing to all slaughtered cattle older than 24 months.

The OTM rule has an empirical basis. Although there have been some 50 reported cases of BSE in animals younger than 30 months, $99.97 \%$ of the roughly 180,000 BSE cases recorded in Europe since 1986 - the vast majority of them in Britain — have fallen into this age group. In Britain, consumption of OTM cattle is banned.

The tests approved by the EU have subtle differences, but all use antibodies to detect the 'prions' that accumulate in the brains of cattle with BSE. These rogue proteins are thought to cause the disease by converting a protein called PrP into their own, misshapen form - known as $\operatorname{PrP}^{\mathrm{Sc}}$ or $\mathrm{PrP}^{\mathrm{res}}$. In 1999, Jim Moynagh of the European Commission's Consumer Policy and Health Protection Directorate General and Heinz Schimmel of the Institute for Reference Materials and Measurements in Geel, Belgium, subjected four diagnostic tests to validation studies. Each was put through its paces on 300 samples of brain tissue from cows with BSE and 1,000 samples from healthy New Zealand cows. Three of the tests - made by Prionics of Zurich, Enfer Scientific in Tipperary, Ireland, and the French Atomic Energy Commission (CEA) - emerged with a 100\% record, recording neither false negatives nor false positives ${ }^{2}$. In practice, the tests do have a very small error rate when tested on tens of thousands of samples.

But the problem is that this validation did not assess the tests' ability to detect infection 
in cows incubating BSE - which are, after all, those that the EU's testing programme hopes to identify. So far, the best way to detect preclinical BSE infection has been to take a sample of brain tissue from the cow in question and inject it into the brains of mice; if these animals later develop the disease, then the cow was incubating BSE.

Because this bioassay takes many months to run, it is impractical as a working diagnostic tool. But new evidence suggests that at least one of the approved tests is similarly sensitive. The CEA test, produced commercially by BioRad of Hercules, California, was recently tested against the mouse bioassay in its ability to detect $\mathrm{PrP}^{\mathrm{Sc}}$ in diluted samples of brain from cattle with BSE. The results, published last month $^{3}$, revealed that Bio-Rad's test and the mouse bioassay performed equally well, even on samples diluted to one in 1,000. "The results were at least as accurate as those provided by the mouse bioassay," says Moynagh.

"This looks like a very good test," agrees Paul Brown, a prion disease researcher at the US National Institute of Neurological Disorders and Stroke in Bethesda, Maryland. Nonetheless, its ability to perform well with diluted samples from BSE cases does not prove that the test will reliably identify preclinical BSE. "The whole testing programme is a waste of time if tests cannot detect preclinical disease well enough," Brown says.

\section{Deliberately infected}

Providing the evidence that Brown wants to see will require the tests to be validated on tissues from cattle that have been deliberately infected. Only then will it be possible to determine whether any test can provide a preclinical diagnosis; and, if so, to determine the point during the course of infection at which diagnosis becomes reliable.

Just one laboratory can provide the necessary samples. In 1998, at Britain's Central Veterinary Laboratory in Weybridge, Surrey, 200 calves were infected with BSE at two different doses. At three-month intervals, groups of four of them, plus two healthy controls, are slaughtered and samples of their fluids and tissues are harvested and frozen.

This followed from an earlier study, in which 30 cattle were infected with BSE and then slaughtered at regular intervals to investigate, using the mouse bioassay, which tissues might pose a risk to human health, and to determine when they become infective 4 . Samples from the first study have all been used up. But Gerald Wells, the veterinary pathologist in charge of both studies, says that scientists who want access to frozen samples from the ongoing study to validate diagnostic tests are welcome to make requests. "If people want preclinical tissue," he says, "then we can make it available."

If the tests currently in use can reliably detect preclinical BSE, then the EU's testing programme should prove invaluable to epi- demiologists striving to analyse the dynamics of the epidemic.

Using incomplete data, epidemiologists have already made some progress. Based on the available French data, for instance, Christl Donnelly of London's Imperial College last year estimated that since 1987 at least 1,200 French cattle have been infected with BSE ${ }^{5}$. Similar analyses are possible for countries such as Switzerland, which has instituted its own programme of BSE testing. But for many European countries, epidemiologists cannot begin to bring their skills to bear. "Estimations are practically impossible if there are only a few reported cases," says Donnelly.

The current generation of diagnostic tests may prove to be of limited value in detecting preclinical cases. In any case, it would be much better to have a simple blood test that could be used on live animals, rather than one that requires samples of brain tissue. In that regard, some experts are encouraged by the discovery last year that prions bind to a blood protein called plasminogen ${ }^{6}-$ an interaction that might be used to develop a blood test. Others point to the work of Mary Jo Schmerr

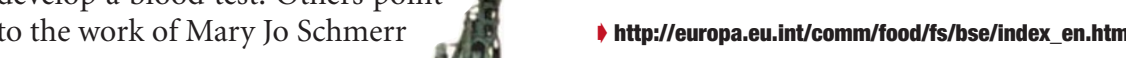

of the National Animal Disease Center in Ames, Iowa, who has shown that it is possible to detect the tiny quantities of prions in the blood of sheep infected with scrapie, a related disease ${ }^{7}$. In this test, the prions compete with fluorescently labelled synthetic PrP proteins to bind to antibodies, and the resulting mixture is separated and analysed by being passed through a capillary.

Whether or not the answers can come from current diagnostic tests, experts agree that thorough testing of its cattle herds offers the best route for Europe to get to grips with its BSE problem. "We want to understand how the epidemic behaves," says Adriano Aguzzi, a neuropathologist at the University of Zurich. "Since we estimate a massive number of unreported cases in many countries, more testing is the only way to find out."

Quirin Schiermeier is Nature's German correspondent.

1. Butler, D. Nature 381, 544-545 (1996).

2. Moynagh, J. \& Schimmel, H. Nature 400, 105 (1999).

3. Deslys, J. P. et al. Nature 409, 476-478 (2001).

4. Wells, G. A. H. et al. Vet. Rec. 148, 103-106 (1998).

5. Donnelly, C. Nature 408, 787-788 (2000).

6. Fischer, M. B. et al. Nature 408, 479-483 (2000).

Schmerr, M. J. et al. J. Chromatogr. A 853, 207-214 (1999).

Requiem for a herd: dumped because they can no longer be sold, these cows in northern Spain await incineration.
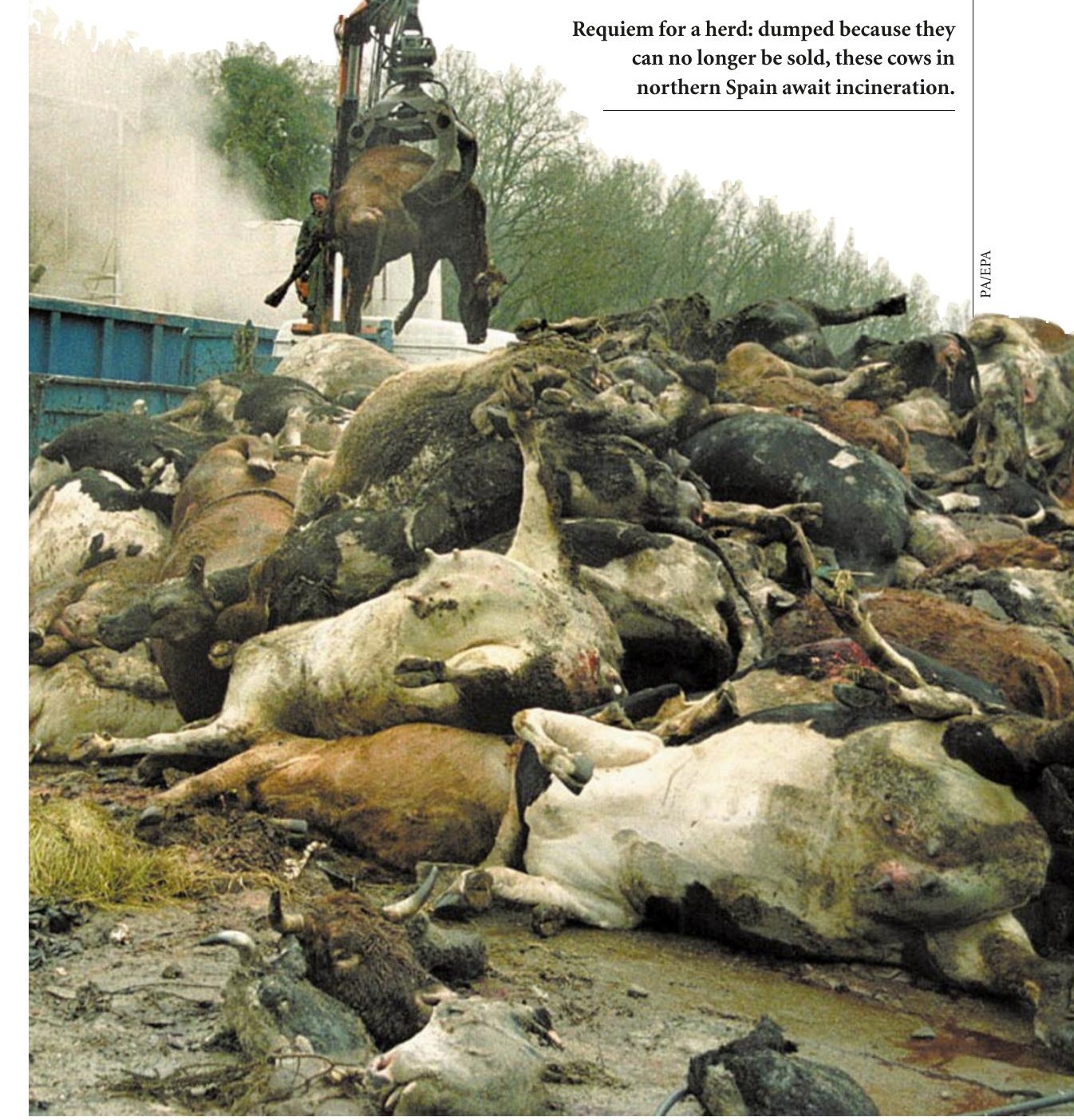